\title{
Involvement of the Spinal Cord in Primary Mitochondrial Disorders: A Neuroimaging Mimicker of Inflammation and Ischemia in Children
}

\author{
(D) C.A.P.F. Alves, (D) A. Goldstein, (D) S.R. Teixeira, (D).S. Martin-Saavedra, (DI.P. de Barcelos, (D) G. Fadda, (D). Caschera, (D) M. Kidd, \\ (D)F.G. Gonçalves, (D)E.M. McCormick, (D).J. Falk, (D) Z. Zolkipli-Cunningham, (D)A. Vossough, and (D). Zuccoli
}

\begin{abstract}
BACKGROUND AND PURPOSE: Little is known about imaging features of spinal cord lesions in mitochondrial disorders. The aim of this research was to assess the frequency, imaging features, and pathogenic variants causing primary mitochondrial disease in children with spinal cord lesions.

MATERIALS AND METHODS: This retrospective analysis included patients seen at Children's Hospital of Philadelphia between 2000 and 2019 who had a confirmed diagnosis of a primary (genetic-based) mitochondrial disease and available MR imaging of the spine. The MR imaging included at least both sagittal and axial fast spin-echo T2-weighted images. Spine images were independently reviewed by 2 neuroradiologists. Location and imaging features of spinal cord lesions were correlated and tested using the Fisher exact test.

RESULTS: Of 119 children with primary mitochondrial disease in whom MR imaging was available, only 33 of 119 (28\%) had available spine imaging for reanalysis. Nineteen of these 33 individuals (58\%) had evidence of spinal cord lesions. Two main patterns of spinal cord lesions were identified: group A $(12 / 19 ; 63 \%)$ had white \pm gray matter involvement, and group B $(7 / 19 ; 37 \%)$ had isolated gray matter involvement. Group A spinal cord lesions were similar to those seen in patients with neuromyelitis optica spectrum disorder, multiple sclerosis, anti-myelin oligodendrocyte glycoprotein-IgG antibody disease, and leukoencephalopathy with brain stem and spinal cord involvement and lactate elevation. Group B patients had spinal cord findings similar to those that occur with ischemia and viral infections. Significant associations were seen between the pattern of lesions (group A versus group B) and the location of lesions in cervical versus thoracolumbar segments, respectively $(P<.01)$.
\end{abstract}

CONCLUSIONS: Spinal cord lesions are frequently observed in children with primary mitochondrial disease and may mimic more common causes such as demyelination and ischemia.

ABBREVIATIONS: mtDNA = mitochondrial DNA; NMOSD = neuromyelitis optica spectrum disorder; LBSL = leukoencephalopathy with brain stem and spinal cord involvement and lactate elevation; MOG = myelin oligodendrocyte glycoprotein; LHON = Leber hereditary optic neuropathy; AQP4-Ab = antiaquaporin 4 antibody

rimary mitochondrial disorders are a heterogeneous group of genetic diseases that can present in childhood and cause

Received May 26, 2020; accepted after revision September 25.

From the Division of Neuroradiology, Department of Radiology (C.A.P.F.A., S.R.T., J.S.M.S., L.C., F.G.G, A.V., G.Z.), Division of Human Genetics, Department of Pediatrics (A.G., E.M.M., M.J.F., Z.Z.-C.), Mitochondrial Medicine Frontier Program, Division of Human Genetics (I. P.d.B.), Department of Pediatrics, Center for Applied Genomics, Children's Hospital of Philadelphia, Philadelphia, Pennsylvania; Departments of Neurology (G.F.) and Pediatrics (A.G., M.J.F., Z.Z.-C.), University of Pennsylvania Perelman School of Medicine, Philadelphia, Pennsylvania; Centre for Statistical Consultation (M.K.), University of Stellenbosch, Stellenbosch, South Africa; The Program for the Study of Neurodevelopment in Rare Disorders (G.Z.), Children's Hospital of Pittsburgh of UPMC, Pittsburgh, Pennsylvania; and Neuroradiology Unit (L.C.), Fondazione IRCCS Ca' Granda Ospedale Maggiore Policlinico, Via Francesco Sforza 35, Milan, Italy

This work was funded in part by The CHOP Mitochondrial Medicine Frontier Program and the National Institutes of Health (U24-HD093483 to M.J.F.) as the sources of research funding. The Institutional Development Fund (IDF) from the CHOP Center for Applied Genomics (CAG) supported I.-P.d.B's work. The content is solely the responsibility of the authors and does not necessarily represent the official views of the National Institutes of Health.

multisystemic impairment, with particular involvement of the CNS. ${ }^{1}$ Little is known about spinal cord lesions in individuals with mitochondrial disorders despite the high frequency of brain involvement and the several well-described cerebral imaging patterns. A recent review of original articles published between 1966 and 2017 focused on spinal cord findings in patients with mitochondrial disorders, concluding that the spinal cord may be involved in various mitochondrial disorders. ${ }^{2}$ However, the different imaging patterns and the frequency of spinal cord lesions in these patients remain poorly defined. One exception is the

Please address correspondence to C.A.P.F. Alves, Division of Neuroradiology, Department of Radiology, Children's Hospital of Philadelphia, Philadelphia, PA; e-mail: alvesc@email.chop.edu

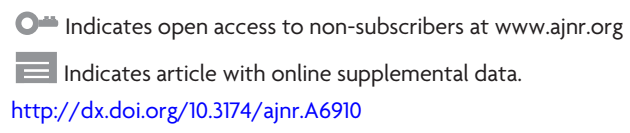

AJNR Am J Neuroradiol 42:389-96 Feb 2021 www.ajnr.org 
well-described spinal cord involvement that occurs in individuals with leukoencephalopathy with brain stem and spinal cord involvement and lactate elevation (LBSL) that is caused by pathogenic variants in DARS2, which is characterized by cervical spinal lesions affecting the posterior columns and lateral corticospinal tracts. $^{3,4}$

Common mitochondrial disease phenotypes such as Leigh syndrome, Kearns-Sayre syndrome, and POLG (polymerase gamma)-related disorders and other pathogenic gene disorders have not had spinal cord imaging results described, with the exception of several small case reports and neuropathologic studies. ${ }^{5,6}$ To address this knowledge deficiency, the present study aimed to assess the frequency, MR imaging features, and clinical and genetic associations of spinal cord lesions that occur in children with primary (genetic-based) mitochondrial disease.

\section{MATERIALS AND METHODS Setting and Participants}

This retrospective study was reported according to the Strengthening the Reporting of Observational Studies in Epidemiology statement. ${ }^{7}$ The study was conducted under an approval by the institutional review board of The Children's Hospital of Philadelphia (CHOP) \#18-015488. Clinical cases were identified from the Mitochondrial Medicine Frontier Program in IRB-approved CHOP Study \#08-6177 (M.J.F., Principal Investigator) and by electronic medical record data base search of brain MR imaging reports spanning January 2000 to January 2019 using the keywords "mitochondrial disease" or "mitochondrial disorder."

Included individuals had a genetically confirmed primary mitochondrial disorder identified by mitochondrial DNA sequencing, nuclear gene panel-based testing, or exome sequencing, with inheritance pattern confirmed when parental samples were available, and had undergone spinal MR imaging, with images available for review of at least the cervical segment. Patients older than 18 years of age at clinical onset and those with low-quality images were excluded. To avoid classification bias, 1 pediatric neurologist who specializes in mitochondrial disorders and 1 genetic counselor (20 and 8 years' clinical experience, respectively) reviewed medical records to confirm pathogenic genetic variants (mutations), clinical diagnosis, and the accuracy of the collected clinical data.

\section{Collected Data}

Clinical data were abstracted from the medical record for age at disease onset, sex, clinical presentation at onset, age and clinical presentation at time of imaging, and genotypes. Children were classified according to pathogenic variant, mitochondrial (mtDNA) or nuclear gene disorder, and mitochondrial pathway functional classifications. ${ }^{1}$ Neurologic symptoms present at the time of spinal cord imaging study were recorded, as well as the results of testing for CSF oligoclonal bands, serum antiaquaporin 4 antibody (AQP4$\mathrm{Ab}$ ), and antimyelin oligodendrocyte glycoprotein antibodies (antiMOG), when available.

Spine MR imaging, previously performed for clinical purposes, was independently reviewed by 2 neuroradiologists with more than 5 years of experience in pediatric neuroradiology. Disagreements

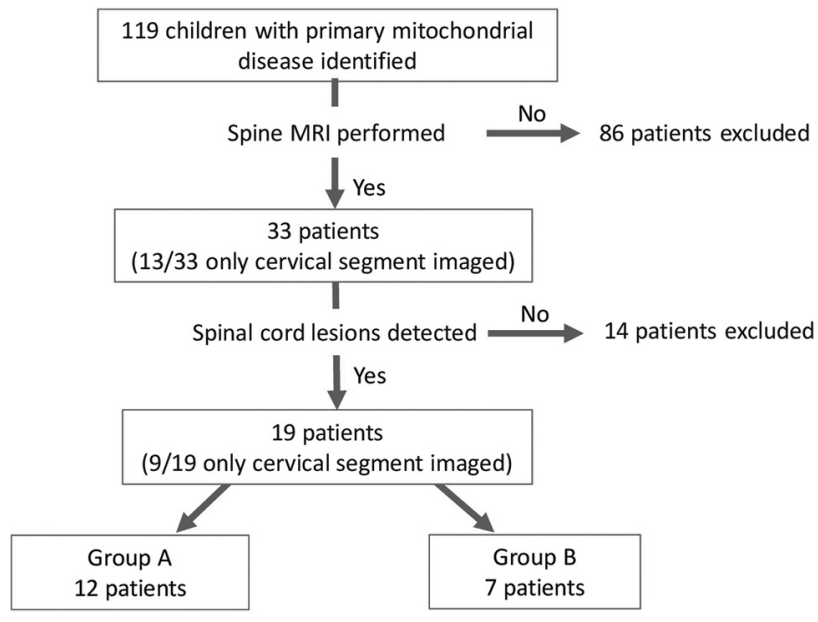

FIG 1. Flowchart of patient selection and inclusion criteria for our cohort.

were resolved by a third pediatric neuroradiologist ( 18 years of experience). MR imaging was done on a variety of $1.5 \mathrm{~T}$ and $3 \mathrm{~T}$ scanners (Siemens, GE Healthcare, Philips Healthcare). Studies included at least sagittal and axial fast spin-echo T2-weighted MR imaging: Axial T2-section thickness $=3-5 \mathrm{~mm}$, section gap $=$ $0-2.5 \mathrm{~mm}, \mathrm{TR}=2616-5720 \mathrm{~ms}, \mathrm{TE}=92-114 \mathrm{~ms}$, flip angle $=$ $90-160^{\circ}$, echo-train length $=14-29$, acquisition matrix $=156-$ $320 \times 108-240$; Sagittal T2 - section thickness $=3 \mathrm{~mm}$, section gap $=0-0.3 \mathrm{~mm}, \mathrm{TR}=2500-5150 \mathrm{~ms}, \mathrm{TE}=81-113 \mathrm{~ms}$, flip angle $=90-180^{\circ}$, echo-train length $=16-35$, acquisition matrix $=184-448 \times 134-336$.

MR imaging examinations were reviewed on a PACS and data were collected using a standardized form. Reviewers were blinded to patients' genetic diagnoses and clinical phenotypes.

Spinal cord MR imaging abnormalities were described according to the spinal cord segment involved (cervical, thoracolumbar, or both), their longitudinal extent (long if $\geq 3$ vertebral bodies, or focal if $<3$ vertebral bodies), and the transverse distribution of the lesions (central, anterior, posterior, lateral columns, or whole cross-section). Spinal cord lesions were further classified into 2 main imaging categories, with group A having involvement of white matter (or both white and gray matter) and group B having exclusive involvement of gray matter.

\section{Statistical Analysis}

Data were analyzed using Statistica, version 13.5 (TIBCO Data Science). Numeric variables were described according to their distribution, with median and IQR. Categoric variables were expressed as percentage and frequency. Associations of the location of lesions with patterns of the lesion (group A versus group B) were tested using the Fisher exact test. Statistical significance was defined as $P<.05$.

\section{RESULTS}

Among 119 children identified with genetically confirmed primary mitochondrial disease who underwent CNS MR imaging studies, 33 had spine MR imaging performed of at least the cervical segment. Twenty of these 33 patients had MR imaging 


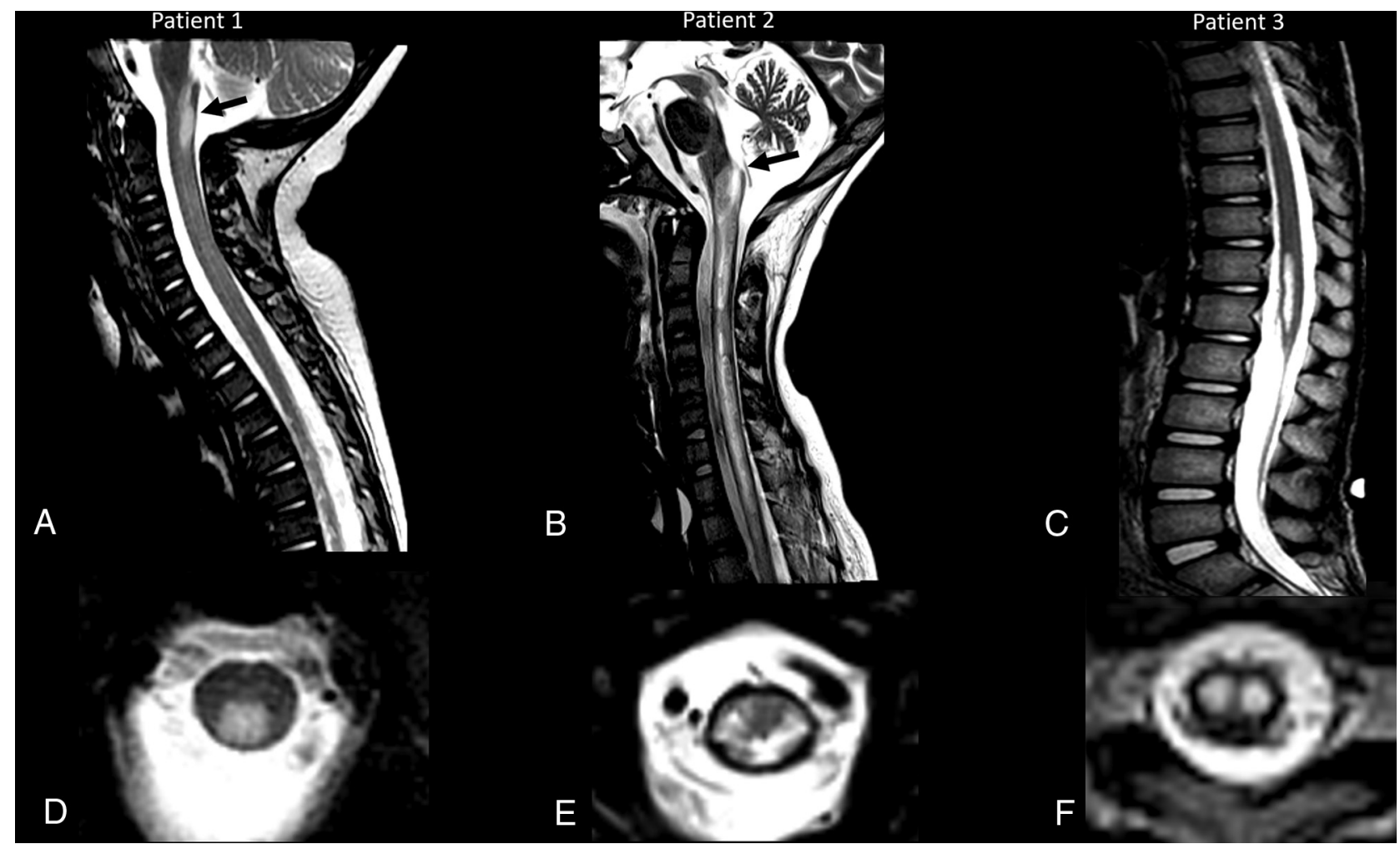

FIG 2. Segment distribution of the lesions and different appearances. Three patients with MR imaging spinal cord lesions in different segments of the spinal cord. Imaging in patient 1 ( $A$ and $D$ ) with an NDUFS1 pathogenic variant (c.365C > T:p.Prol22Leu and c.155 + 1G >A) shows a lesion located in the cervical segment (arrow, $A$ ), at the level of $\mathrm{Cl}$, with a posterior and central distribution on the axial view $(D)$ and extension to the area postrema. Imaging in patient 2 ( $B$ and $E$ ) with MT-ND4 pathogenic variant (m.11777C $>A$ ) shows a spinal cord lesion with diffuse cross-sectional involvement $(E)$, longitudinally extensive, and involving both cervical and thoracic levels, including the area postrema (arrow, $B$ ). Imaging in patient 3 ( $C$ and $F$ ) with MT-ND5 pathogenic variant (m.13513G $>A$ ) shows a thoracolumbar lesion, extending to the conus medullaris, with isolated gray matter involvement and snake eyes appearance.

performed of the entire spine. Nineteen $(19 / 119,16 \%$ of all patients with primary mitochondrial disease; $19 / 33,58 \%$ of those with spine MR imaging) demonstrated spinal cord lesions (Fig 1). The median age at onset of clinical symptoms was 1.76 years (IQR $=0.3-12.3$ years), and the male:female ratio was 0.7:1.

Spine MR imaging studies were obtained for a variety of reasons: children demonstrating weakness $(6 / 19,32 \%), 3$ of whom had acute onset respiratory failure and were found to have brain stem lesions extending to the spinal cord on brain MR imaging, changes in gait $(4 / 19,21 \%)$, acute bowel and bladder symptoms $(4 / 19,21 \%)$, scoliosis $(2 / 19,11 \%)$, and extremity tremor $(2 / 19$, $11 \%)$. Spinal myoclonus and vomiting were present in 1 child each $(1 / 19,5 \%)$, with lesions extending to the area postrema (Online Supplemental Data).

Sixteen $(16 / 19,84 \%)$ patients had lesions located at cervical levels, of whom 6 had lesions that extended to thoracic levels. Three (3/10, 30\%) individuals had lesions restricted to the thoracolumbar levels that extended to the conus medullaris (Fig 2). Eight $(8 / 19,42 \%)$ of the spinal cord lesions were longitudinally extensive, and $11(11 / 19,58 \%)$ were focal in appearance. Six children $(6 / 19,32 \%)$ had lesions reaching the area postrema, and $3(3 / 19,16 \%)$ had lesions with a tumefactive appearance (with cord expansion). Clinical, genetic, and main radiologic findings of these cases with spinal cord involvement are summarized in the Online Supplemental Data. Clinical and demographic features of all patients with a spine study are summarized in the Online Supplemental Data.

Involvement of the cross-sectional anatomy of the spinal cord varied. Lesions were most frequently distributed centrally around the central canal $(11 / 19,58 \%)$ followed by lesions involving the posterior columns $(7 / 19,37 \%)$.

Two main imaging patterns of spinal cord lesions were identified. The group A pattern was present in 12 patients (12/19, $63 \%$ ), with involvement of the peripheral white matter with or without involvement of the central gray matter. This imaging pattern resembled that of demyelinating lesions.

Among the group A cohort, we further subdivided the lesions into 3 major subgroups based on their imaging appearance consistent with typical patterns of particular demyelinating disorders: neuromyelitis optica spectrum disorder (NMOSD)-like in 6 children, anti-MOG-like in 2 children, and MS-like in 2 children. In the group A NMOSD-like MR imaging presentation, spinal cord lesions were longitudinally extensive, mostly accompanied by tumefactive effect and involvement of the area postrema (Fig 3). No positive laboratory test results for AQP4-Ab were detected in these patients. Group A patients who had spinal cord lesions with anti-MOG-like imaging features demonstrated lesions that were longitudinally extensive with diffuse cross-sectional involvement, 


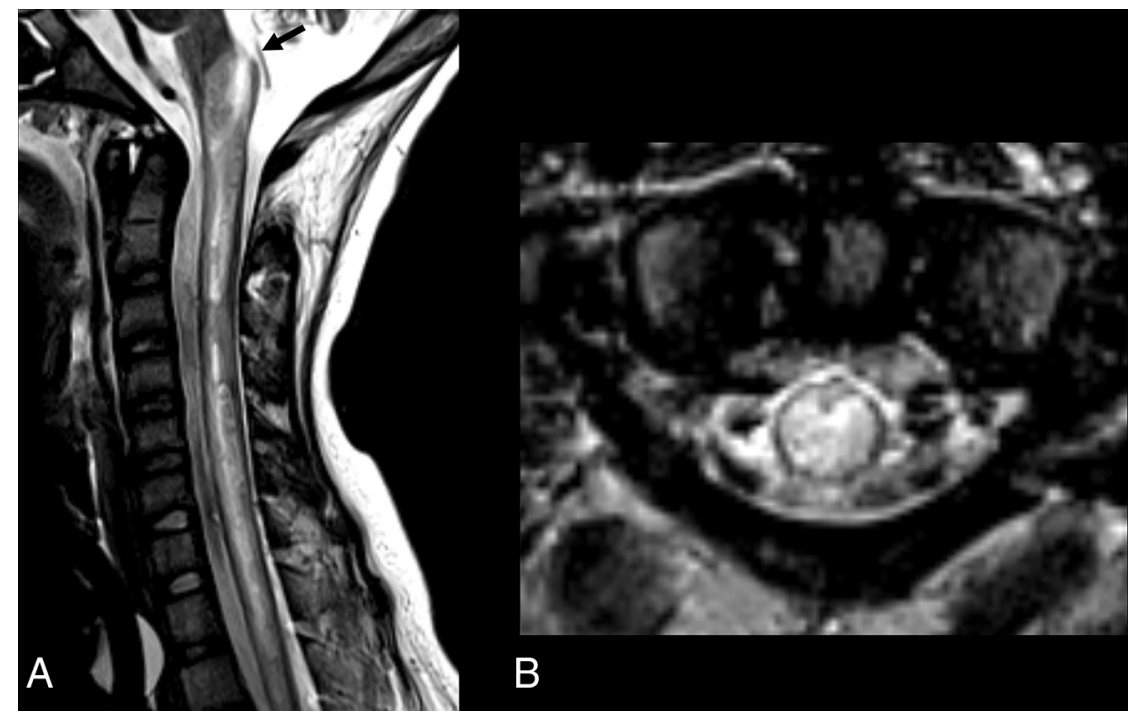

FIG 3. Group A, NMOSD appearance. A 12-year-old male patient with MT-ND4 pathogenic variant $(\mathrm{m} .11777 \mathrm{C}>\mathrm{A})$ with a demyelination-like pattern of the spinal cord lesion and NMOSD-like appearance. Sagittal and axial T2 MR imaging of the cervical spine shows a longitudinal extensive hyperintense lesion in both cervical and thoracic segments with a tumefactive effect and involvement of the area postrema (arrow, $A$ ). The lesion has a diffuse cross-sectional involvement of the cord in the axial plane (B). with more evident abnormalities in the central gray matter giving the "H sign" appearance (Fig 4). Of the 2 patients with anti-MOG-like MR imaging patterns, 1 child with $M T$ TL1 variant was tested for antiMOG antibodies because of a clinical presentation consistent with acute disseminated encephalomyelitis and had positive antibody results, leading to a final diagnosis of anti-MOG antibody associated disease in addition to the primary mitochondrial disorder. The other patient with an anti-MOG-like imaging pattern was not evaluated for the presence of serum antibodies. The 2 children with an MS-like imaging appearance demonstrated numerous lesions that were short in length, located at the cervical and thoracic levels, and distributed within the white matter at the periphery of the cord. No CNS oligoclonal bands were found in these children.

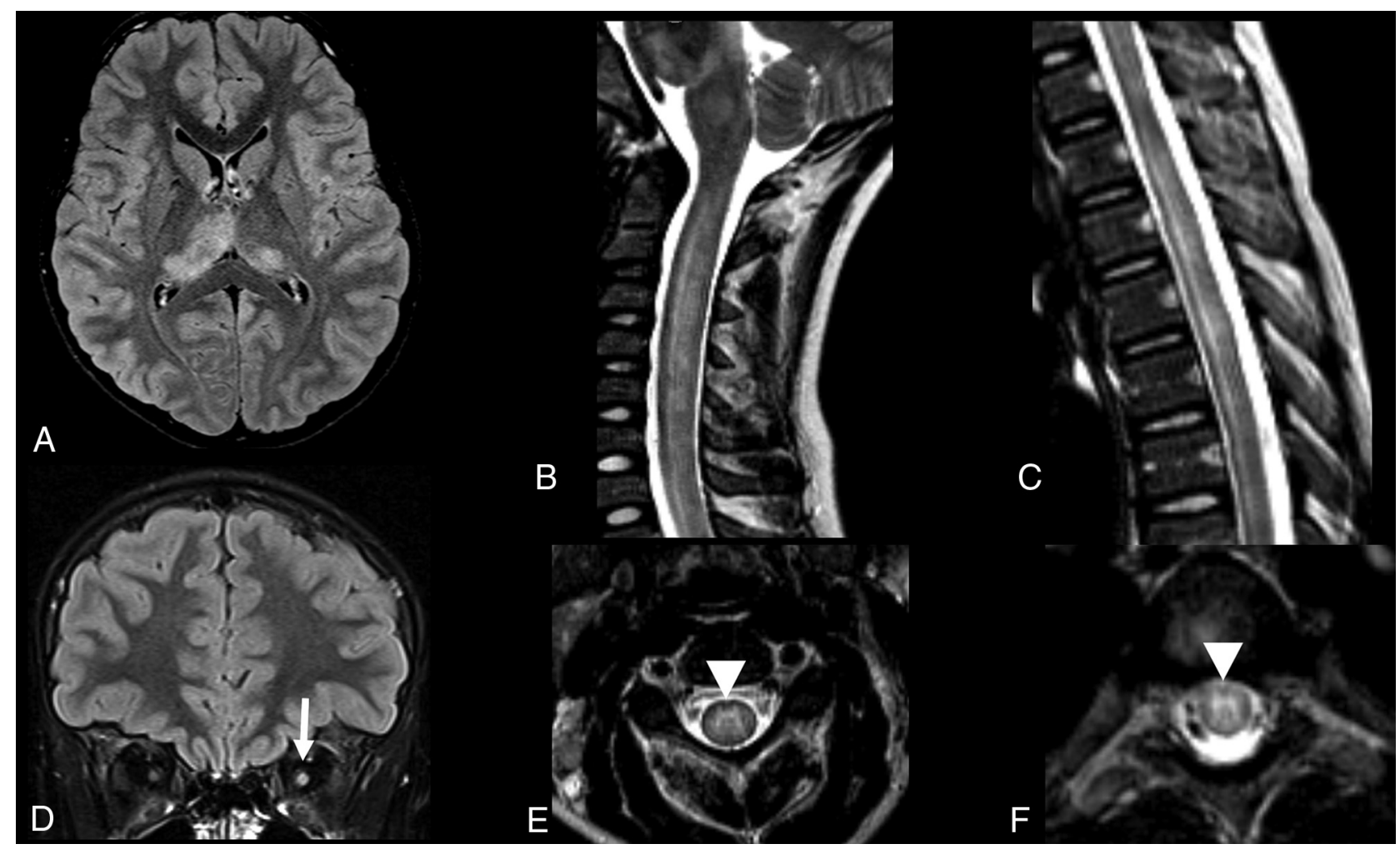

FIG 4. Group A, anti-MOG appearance. A 10-year-old female patient with a family diagnosis of LHON Leber's hereditary optic neuropathy (heteroplasmic m.14484T $>C$ and homoplasmic m.15256G $>$ A mtDNA variants) and MOG + demyelination. Brain MR imaging study axial and coronal FLAIR sequences show asymmetric hyperintense lesions in the thalami, more evident on the right side $(A)$, and enlargement and hyperintensity of the intraorbital left optic nerve (arrow, $D)$. Sagittal and axial spinal MR imaging of the cervical $(B$ and $E)$ and thoracic segments $(C$ and $F$ ) show longitudinal extensive hyperintense lesions in both segments, with more evident involvement of the central gray matter, giving the "H sign" appearance on the axial planes (arrowheads, $E$ and $F$ ). 


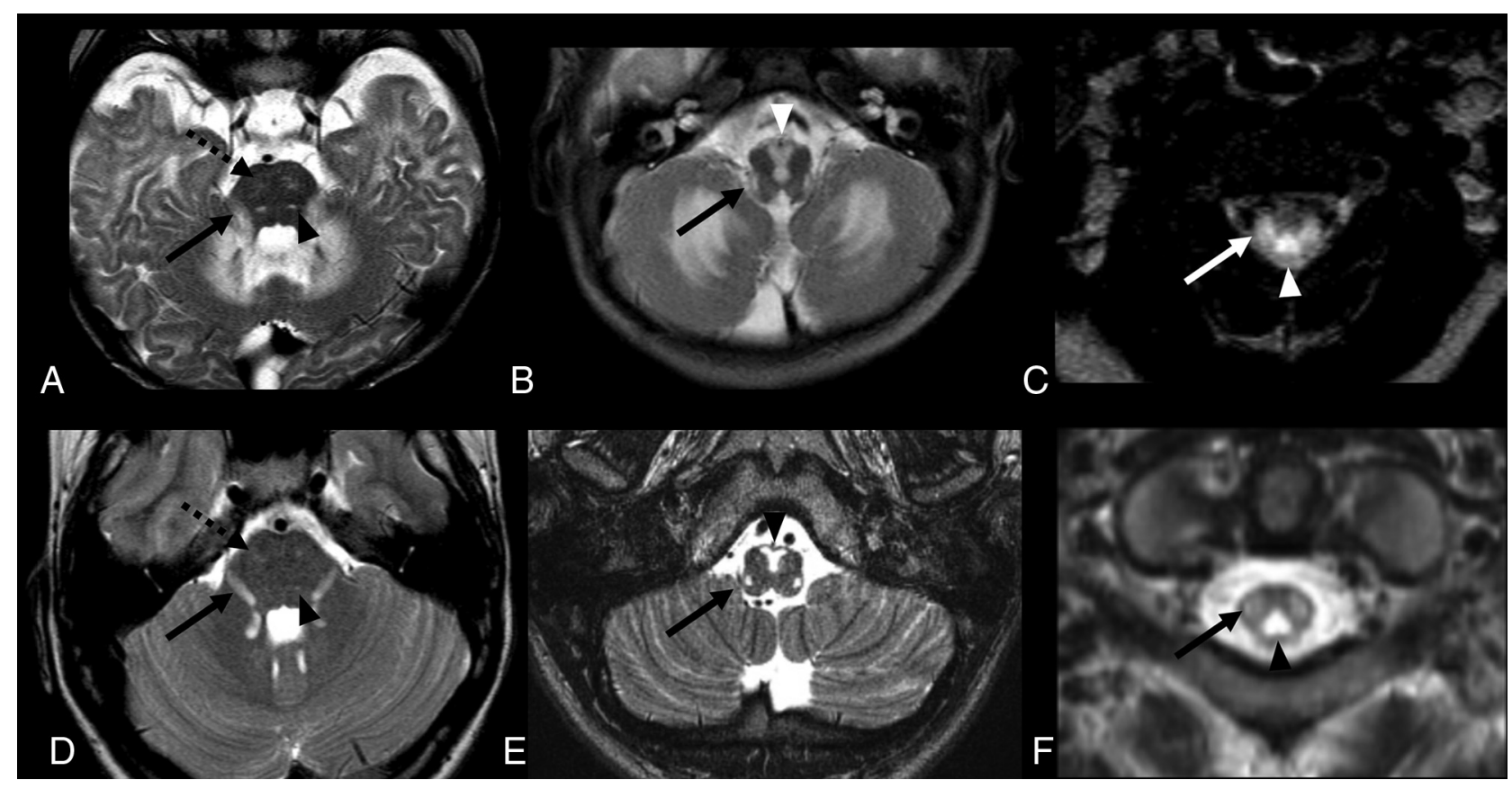

FIG 5. Group A, LBSL appearance. MR imaging of 2 patients with leukoencephalopathy with brain stem and spinal cord involvement and increased lactate (LBSL) appearance. Patient $1(A-C)$ is a 6-month-old male patient with an NDUFS1 pathogenic variant. Patient 2 (D-F) is a 1-yearold male patient with a DARS2 pathogenic variant. In both patients, axial T2 sequences show symmetric hyperintense lesions, affecting both corticospinal tracts, in the pons (dotted arrows, $A$ and $D$ ). Hyperintense lesions are also observed along the intraparenchymal portions of the trigeminal nerves (arrows, $A$ and $D$ ) and medial lemnisci (arrowheads, $A$ and $D$ ). Lesions affecting the pyramids (arrowheads, $B$ and $E$ ) and inferior cerebellar peduncles (arrows, $B$ and $E$ ) are observed in the medulla.On axial T2-weighted cervical spinal MR imaging $(C$ and $F$ ), hyperintense lesions are seen in the dorsal columns (arrowheads) and lateral corticospinal tracts (arrows).

Moreover, 2 patients who were included in group A and designated to have selective white matter involvement presented with imaging features of LBSL. These lesions involved the posterior and lateral columns of the spinal cord in the cervical segment. One of these children had pathogenic variants in DARS2, the commonly known cause of this phenotype, but the other child had NDUFS1 pathogenic variants that were not previously associated with this imaging phenotype. The brain MR imaging of these 2 patients was consistent with the imaging phenotype of LBSL, showing symmetric lesions affecting the bilateral corticospinal tracts, the intraparenchymal part of the trigeminal nerves, and the medial lemnisci (Fig 5).

The group B pattern was observed in 7 patients $(7 / 19,37 \%)$ with lesions limited to the spinal cord gray matter, resembling imaging features of spinal cord infarct or acute viral infections.

Regarding the clinical symptoms, whereas children in group A often demonstrated acute symptoms, children in group B showed gradually progressive symptoms, except for bowel and bladder changes that were always acute. A significant association was found between the pattern of lesions (group A versus group B) and lesions involving the cervical versus thoracolumbar segments $(P<.01)$. Cervical lesions were more frequently observed in group A $(12 / 12$, $100 \%)$ as opposed to group B $(4 / 7,57 \%)$. Thoracolumbar lesions extending to the conus medullaris were observed in group $\mathrm{B}(3 / 7$, $43 \%)$ but not in group A (0/12, $0 \%)$.

\section{DISCUSSION}

Although a few publications have reported spinal cord lesions may occur in very select mitochondrial disorders, limited evaluation has been performed to characterize correlations between spinal cord imaging findings and clinical phenotypes in a broad range of patients with primary mitochondrial disease with well-defined pathogenic causes. ${ }^{3,8-14}$ Here, we performed a comprehensive retrospective review of all spine imaging in patients with confirmed primary mitochondrial disease over the past 2 decades, which has clearly demonstrated that a high frequency of spinal cord lesions occurs in children with primary mitochondrial disorders. Indeed, spinal cord lesions were observed in more than half of our pediatric patients with primary mitochondrial disease who underwent spine imaging.

A detailed description of the histopathologic features of spinal cord lesions in mitochondrial disorders has shown damage of motor, sensory, and autonomic fibers. ${ }^{2,3,6,8-10,15,16}$ Moreover, a variety of findings, including the presence of ischemia, necrosis, relative sparing of neurons, edema of the gray matter, vascular congestion, and demyelination around the central canal and dorsal columns of the cervical cord, have been reported in the neuropathologic literature. ${ }^{5,17}$ These data support our study's findings that imaging features resemble different forms of pediatric demyelination and that more frequent distribution of lesions occurs around the central canal and in the posterior columns of the spinal cord.

Studying the broad spectrum of imaging findings and patterns, we were able to classify the imaging appearance of the spinal cord lesions into 2 distinct patient subtypes, group A and group B. Group A patterns included individuals with overlapping imaging features of lesions observed in demyelinating disorders. 
These lesions tend to be distributed in the cervical and thoracic segments and were restricted to the white matter or affected both white and gray matter. Group A patterns were present in half of the patients with imaging findings typical of NMOSD, ${ }^{18}$ including tumefactive effect, longitudinal extensive lesions, and involvement of the area postrema. These patients were clinically evaluated for demyelinating diseases but with negative results for AQP4-Ab. Possible associations linking NMOSD and mitochondrial disorders have been considered for many years, mainly based on a few patients who demonstrated imaging similarities of NMOSD in the context of having a diagnosis of Leber hereditary optic neuropathy (LHON). ${ }^{19,20}$ As one example, Hudson et $\mathrm{al}^{21}$ sequenced the mtDNA genes commonly known to cause LHON in patients with NMOSD, in addition to specific mtDNA haplogroups that are preferentially associated with pathogenic variants in complex I mtDNA gene that cause LHON, in both patients with NMOSD and control participants. The authors did not find any statistically significant association between these 2 disorders. ${ }^{21}$

However, in recent literature, mtDNA has been increasingly recognized as an agonist of the innate immune system. Its role in inflammasome activation as a damage-associated molecular pattern is shown by its ability to induce the secretion of interleukin- $1 \beta$ from microglia. ${ }^{22,23}$ Interestingly, levels of CSF mtDNA have been noted to be higher in NMOSD AQP4-Ab-positive patients compared with those with other neurologic disorders. Moreover, transfected cells that express AQP4 when incubated with serum from NMOSD AQ4-Ab-positive patients were shown to release mtDNA. ${ }^{24}$ NMOSD AQP4-Ab-negative patients may show similar imaging findings, including spinal cord features resembling positive AQP4-Ab. ${ }^{18}$ Furthermore, primary mitochondrial dysfunction may induce microglial activation. Microglial cells, in turn, can release reactive oxygen species and proinflammatory cytokines, which are able to damage essential components of the mitochondria such as mtDNA. ${ }^{25}$

We postulate that the mtDNA insult and release may trigger neuroinflammation in predisposed individuals, as exemplified by specific case observations. Interestingly, 2 patients in group A who had lesions affecting the whole cross-section of the spinal cord that involved most pronounced signal abnormality in the central gray matter to give the " $\mathrm{H}$ sign" appearance were suspected in their differential diagnosis of having anti-MOG demyelination. ${ }^{26}$ Indeed, 1 of these children harbored a heteroplasmic m.14484T $>$ C known pathogenic variant and positive family history of LHON and had a confirmed anti-MOG Ab diagnosis. Although the spinal cord imaging features for the other patient were compatible for anti-MOG Ab phenotype, this child was not tested because there had been no other supporting clinical indication.

Two children had short-length spinal cord lesions that occurred in the periphery of the cord that involved only the white matter in the cervical and thoracic segments, mimicking MS. However, neither the brain imaging findings nor the laboratory results supported the imaging-based diagnosis of $\mathrm{MS}^{27}$ Reports from the scientific literature highlight the difficulty of a clinical approach to patients affected by mitochondrial disorders who also present with brain lesions resembling demyelination. ${ }^{28,29} \mathrm{We}$ believe that our study's focus on spinal cord MR imaging findings further supports the hypothesis of the mitochondrial cause of these MS-like cases, which also present with overlapping imaging features of autoimmunity.

Two other group A children from our cohort presented with the typical imaging findings of LBSL (following previously established features). ${ }^{8}$ These included 1 child with DARS2 pathogenic variants (a gene that encodes mitochondrial aspartyl-tRNA synthetase described in association with this imaging pattern $)^{8}$ and the other with NDUFS1 pathogenic variants, a nuclear gene encoding a mitochondrial complex I subunit that has not previously been associated with this unique imaging phenotype. Further imaging characterization of larger prospective cohorts affected by these 2 pathogenic genes and related gene classes may be helpful to differentiate and optimize care for individuals with these genetic disorders.

The children in group B included patients who presented with features typical of spinal cord infarct, with all lesions localized within the gray matter. Even though other disorders, such as acute flaccid myelitis caused by viral infection, may present with similar findings, ${ }^{30}$ there is indirect evidence that mitochondrial dysfunction that impairs cellular energy production may be a causal or contributing factor to ischemic tissue changes. ${ }^{31}$ Thus, we believe that ischemic lesions remain the primary pathogenic cause of isolated gray matter spinal cord lesions and accordingly should be excluded in this context. Indeed, some of the ischemic lesions that we identified in children with primary mitochondrial disease occurred within the lower thoracolumbar segment, extended to the conus medullaris, and had the classic ischemic "snake-eye" appearance. ${ }^{32}$

Upon review of clinical phenotypes in our cohort with spinal cord lesions, Leigh syndrome was the most frequently observed; it was present in three-quarters of our cohort. Leigh syndrome cases with spinal cord lesions had diverse gene causes in both genomes, a range of pathogenic variants and lesions that could be grouped into both spinal cord imaging patterns (groups A and B).

Patients with primary mitochondrial disease and spinal cord lesions may present with several different neurologic symptoms, such as monoparesis, spasticity, autonomic dysfunction, or sensory or motor transverse syndrome. Less frequently, asymptomatic cases may occur. Spinal deformities are also commonly observed. ${ }^{2}$ However, it has not previously been clear in the literature whether these neurologic symptoms are sufficiently common to support establishment of a standard MR imaging protocol or interval to routinely analyze the spinal segment for patients with primary mitochondrial disease. In our study, the most common clinical symptoms that led to performing spine MR imaging or brain MR imaging with subsequent spine evaluation (because of lesions depicted on brain imaging in the upper spine) were acute or progressive weakness and respiratory failure. Acute bowel or bladder incontinence or retention, change in gait, neuromuscular scoliosis, hyperreflexia, and myoclonus were also neurologic symptoms observed in our cohort. Moreover, individuals with acute weakness had a higher frequency of group A spinal cord lesions identified both at the cervical level and with longitudinally 
extensive lesions. These data provide strong support to consider updating imaging protocols for children with mitochondrial disease when they have concerning neurologic manifestations. Further study will be needed to determine the potential utility of obtaining spinal MR imaging in patients with definite primary mitochondrial disease before the onset of severe neurologic evidence of spinal cord involvement.

The only statistically significant association observed was between the location of the lesions and the cross-sectional imaging pattern, with lesions involving the cervical segment more frequently observed in group A (demyelination features, white \pm gray matter involvement) and lower segment lesions (thoracic or thoracolumbar) more often observed in group B (ischemic features, selective gray matter involvement).

Ascertainment bias is a potential limitation of our study to estimate the frequency of spinal cord lesions in pediatric patients with primary mitochondrial disease because most individuals who had brain $\mathrm{MR}$ imaging performed during the 2 decades of the study period did not undergo full spine MR imaging. Indeed, all children evaluated in this study had MR imaging performed for at least part of the spinal cord because of pronounced neurologic signs or symptoms indicative of potential spinal cord involvement. Retrospective analysis of spinal cord images in this symptomatic cohort demonstrated the relatively high frequency of and value of clinical spine MR imaging to identify spinal cord lesions in patients with primary mitochondrial disorders who have concerning neurologic presentations. Despite the relatively low number of patients with definite mitochondrial disease who had spine MR imaging available for review, this study is the largest cohort reported to date and clearly demonstrates that spinal cord lesions are not an infrequent finding in children with a diverse array of primary mitochondrial diseases.

Furthermore, these analyses discovered that 2 main imaging patterns for spinal cord lesions occur in the context of primary mitochondrial disease, which may help to broaden the differential diagnosis of other common entities such as demyelination, ischemic, or viral disorders.

\section{CONCLUSIONS}

Spinal cord lesions are common in children with primary mitochondrial disorder who manifest with neurologic signs and symptoms. Spine imaging should be considered if spinal cord involvement is suspected based on the clinical associations described in this report. Moreover, spinal cord lesions related patients with primary mitochondrial disease may mimic demyelination, with features that overlap with those of NMOSD, anti-MOG antibody-associated demyelination, or MS, with a possible cervical predilection (group A, white \pm gray matter involvement), or they may mimic ischemia, with the classic snake-eye appearance and may have a predilection along the lower segments of the spinal cord (group B, selective gray matter involvement). The medical community should be aware that primary mitochondrial disorders affect the spinal cord with lesions similar to those observed in cord autoimmunity and ischemia.

\section{ACKNOWLEDGMENTS}

We are grateful to the patients with mitochondrial disease and their families for their involvement in this study. We also thank Lydia Sheldon, MSEd, and Anthony Rosner, $\mathrm{PhD}$, for helpful editing suggestions.

Disclosures: Amy Goldstein—RELATED: Consulting Fee or Honorarium: MitoAction, Comments: Grand rounds/family meeting support group travel fees, directly related to mitochondrial disease; Support for Travel to Meetings for the Study or Other Purposes: UMDF, Wellcome Trust, Comments: Honorarium speaker travel fees directly related to mitochondrial disease. Juan Martin-Saavedra-UNRELATED: Employment: The Children's Hospital of Philadelphia. Giulia Fadda—UNRELATED: Employment: University of Pennsylvania, Comments: I receive salary support from the clinical research training scholarship in multiple sclerosis from the American Academy of Neurology. Martin Kidd-RELATED: Consulting Fee or Honorarium: University of Stellenbosch, South Africa*; UNRELATED: Consultancy: University of Stellenbosch, South Africa*. Marni Falk_UNRELATED: Board Membership: Ribonova Inc, Khondrion, Luciole Pharma; Consultancy: Reneo Pharmaceuticals, Mission Therapeutics, Imel Therapeutics, Cyclerion, Astellas, Neurovive Inc; Patents (Planned, Pending or Issued): Several patents; Royalties: Elsevier; Stock/Stock Options: RiboNova Inc, MitoCUREia; Travel/Accommodations/Meeting Expenses Unrelated to Activities Listed: United Mitochondrial Disease Foundation. Zarazuela ZolkipliCunningham—UNRELATED: Employment: Children's Hospital of Philadelphia. *Money paid to institution.

\section{REFERENCES}

1. Rahman J, Rahman S. Mitochondrial medicine in the omics era. Lancet 2018;391:2560-74 CrossRef Medline

2. Finsterer J, Zarrouk-Mahjoub S. Involvement of the spinal cord in mitochondrial disorders. J Neurosci Rural Pract 2018;9:245-51 CrossRef Medline

3. van der Knaap MS, van der Voorn P, Barkhof F, et al. A new leukoencephalopathy with brainstem and spinal cord involvement and high lactate. Ann Neurol 2003;53:252-58 CrossRef Medline

4. Lin T-K, Chang Y-Y, Lin H-Y, et al. Mitochondrial dysfunctions in leukoencephalopathy with brainstem and spinal cord involvement and lactate elevation (LBSL). PLoS One 2019;14:e0224173 CrossRef Medline

5. Cavanagh JB, Harding BN. Pathogenic factors underlying the lesions in Leigh's disease. Brain 1994;117:1357-76 CrossRef Medline

6. McKelvie P, Marotta R, Thorburn DR, et al. A case of myelopathy, myopathy, peripheral neuropathy and subcortical grey matter degeneration associated with recessive compound heterozygous POLG1 mutations. Neuromuscul Disord 2012;22:401-05 CrossRef Medline

7. von Elm E, Altman DG, Egger M, STROBE Initiative, et al. The Strengthening the Reporting of Observational Studies in Epidemiology (STROBE) statement: guidelines for reporting observational studies. Lancet 2007;370:1453-57 CrossRef Medline

8. Uluc K, Baskan O, Yildirim KA, et al. Leukoencephalopathy with brain stem and spinal cord involvement and high lactate: a genetically proven case with distinct MRI findings. J Neurol Sci 2008;273:118-22 CrossRef Medline

9. Yamashita S, Miyake N, Matsumoto N, et al. Neuropathology of leukoencephalopathy with brainstem and spinal cord involvement and high lactate caused by a homozygous mutation of DARS2. Brain Dev 2013;35:312-16 CrossRef Medline

10. Nikali K, Suomalainen A, Saharinen J, et al. Infantile onset spinocerebellar ataxia is caused by recessive mutations in mitochondrial proteins Twinkle and Twinky. Hum Mol Genet 2005;14:2981-90 CrossRef Medline

11. Bindu PS, Arvinda $\mathrm{H}$, Taly AB, et al. Magnetic resonance imaging correlates of genetically characterized patients with mitochondrial disorders: a study from south India. Mitochondrion 2015;25:6-16 CrossRef Medline

12. Mauri E, Dilena R, Boccazzi A, et al. Subclinical Leber's hereditary optic neuropathy with pediatric acute spinal cord onset: more than meets the eye. BMC Neurol 2018;18:220 CrossRef Medline 
13. Miyauchi A, Osaka H, Nagashima M, et al. Leigh syndrome with spinal cord involvement due to a hemizygous NDUFA1 mutation. Brain Dev 2018;40:498-502 CrossRef Medline

14. Tenney JR, Prada CE, Hopkin RJ, et al. Early spinal cord and brainstem involvement in infantile Leigh syndrome possibly caused by a novel variant. J Child Neurol 2013;28:1681-85 CrossRef Medline

15. Sparaco M, Cavallaro T, Rossi G, et al. Immunohistochemical demonstration of spinal ventral horn cells involvement in a case of "myoclonus epilepsy with ragged red fibers" (MERRF). Clin Neuropathol 2000;19:200-07 Medline

16. Barz H, Henker J. Pathological findings in subacute necrotizing encephalomyelopathy (Leigh's disease) (author's transl). Zentralbl Allg Pathol 1975;119:488-94 Medline

17. Leigh D. Subacute necrotizing encephalomyelopathy in an infant. $J$ Neurol Neurosurg Psychiatry 1951;14:216-21 CrossRef Medline

18. Wingerchuk DM, Banwell B, Bennett JL, et al. International consensus diagnostic criteria for neuromyelitis optica spectrum disorders. Neurology 2015;85:177-89 CrossRef Medline

19. Simão LM. Neuromyelitis optica antibody in Leber hereditary optic neuropathy: case report. Arq Bras Oftalmol 2012;75:280-82 CrossRef Medline

20. McClelland CM, Van Stavern GP, Tselis AC. Leber hereditary optic neuropathy mimicking neuromyelitis optica. J Neuroophthalmol 2011;31:265-68 CrossRef Medline

21. Hudson G, Mowbray C, Elson JL, et al. Does mitochondrial DNA predispose to neuromyelitis optica (Devic's disease)? Brain 2008;131: e93-93 CrossRef Medline

22. Zhang Q, Raoof M, Chen Y, et al. Circulating mitochondrial DAMPs cause inflammatory responses to injury. Nature 2010;464:104-07 CrossRef Medline
23. West AP, Shadel GS. Mitochondrial DNA in innate immune responses and inflammatory pathology. Nat Rev Immunol 2017; 17:363-75 CrossRef Medline

24. Yamashita K, Kinoshita M, Miyamoto K, et al. Cerebrospinal fluid mitochondrial DNA in neuromyelitis optica spectrum disorder. Neuroinflammation 2018;15:125 CrossRef Medline

25. Di Filippo M, Chiasserini D, Tozzi A, et al. Mitochondria and the link between neuroinflammation and neurodegeneration. $J$ Alzheimers Dis 2010;20:S369-79 CrossRef Medline

26. Dubey D, Pittock SJ, Krecke KN, et al. Clinical, radiologic, and prognostic features of myelitis associated with myelin oligodendrocyte glycoprotein autoantibody. JAMA Neurol 2019;76:301-09 CrossRef Medline

27. De Angelis F, Brownlee WJ, Chard DT, et al. New MS diagnostic criteria in practice. Pract Neurol 2019;19:64-67 CrossRef Medline

28. Pfeffer G, Burke A, Yu-Wai-Man P, et al. Clinical features of MS associated with Leber hereditary optic neuropathy mtDNA mutations. Neurology 2013;81:2073-81 CrossRef Medline

29. Bittner F, Falardeau J, Spain RI. Myelin oligodendrocyte glycoprotein antibody-associated demyelination comorbid with Leber hereditary optic neuropathy. JAMA Neurol 2019;76:22728 CrossRef Medline

30. Cheng $\mathrm{H}$, Zeng J, Li H, et al. Neuroimaging of HFMD infected by EV71. Radiol Infect Dis 2015;1:103-08 CrossRef

31. Pathak D, Berthet A, Nakamura K. Energy failure: does it contribute to neurodegeneration? Ann Neurol 2013;74:506-16 CrossRef Medline

32. Vargas MI, Gariani J, Sztajzel R, et al. Spinal cord ischemia: practical imaging tips, pearls, and pitfalls. AJNR Am J Neuroradiol 2015;36:82530 CrossRef Medline 